\title{
Cataclysmic Variables and a New Class of Faint Ultraviolet Stars in the Globular Cluster NGC 6397
}

\section{Citation}

Cool, Adrienne M., Jonathan E. Grindlay, Haldan N. Cohn, Phyllis M. Lugger, and Charles D. Bailyn. 1998. "Cataclysmic Variables and a New Class of Faint Ultraviolet Stars in the Globular Cluster NGC 6397." The Astrophysical Journal 508 (1): L75-79. https://doi.org/10.1086/311730.

\section{Permanent link}

http://nrs.harvard.edu/urn-3:HUL.InstRepos:41399829

\section{Terms of Use}

This article was downloaded from Harvard University's DASH repository, and is made available under the terms and conditions applicable to Other Posted Material, as set forth at http:// nrs.harvard.edu/urn-3:HUL.InstRepos:dash.current.terms-of-use\#LAA

\section{Share Your Story}

The Harvard community has made this article openly available.

Please share how this access benefits you. Submit a story.

Accessibility 


\title{
CATACLYSMIC VARIABLES AND A NEW CLASS OF FAINT ULTRAVIOLET STARS IN THE GLOBULAR CLUSTER NGC $6397^{1}$
}

\author{
Adrienne M. COOL \\ Department of Physics and Astronomy, San Francisco State University, 1600 Holloway Avenue, San Francisco, CA 94132; cool@sfsu.edu \\ JONATHAN E. GRINDLAY \\ Department of Astronomy, Harvard University, 60 Garden Street, Cambridge, MA 01238; josh@cfa.harvard.edu \\ Haldan N. Cohn and Phyllis M. Lugger \\ Department of Astronomy, Indiana University, 319 Swain West, Bloomington, IN 47405; cohn@indiana.edu, lugger@indiana.edu \\ AND \\ Charles D. Bailyn \\ Department of Astronomy, Yale University, P.O. Box 208101, New Haven, CT 06520-8101; bailyn@astro.yale.edu \\ Received 1998 August 24; accepted 1998 September 24; published 1998 October 21
}

\begin{abstract}
We present evidence that the globular cluster NGC 6397 contains two distinct classes of centrally concentrated UV-bright stars. Color-magnitude diagrams constructed from $U_{336}, B_{439}, V_{555}$, and $I_{814}$ data obtained with the Hubble Space Telescope Wide Field Planetary Camera 2 reveal seven UV-bright stars fainter than the main-sequence turnoff, three of which had previously been identified as cataclysmic variables (CVs). Light curves of these stars show the characteristic "flicker" of CVs as well as longer term variability. A fourth star is identified as a CV candidate on the basis of its variability and UV excess. Three additional UV-bright stars show no photometric variability and have broadband colors characteristic of B stars. These nonflickering UV stars are too faint to be extended horizontal-branch stars. We suggest that they could be low-mass helium white dwarfs, formed when the evolution of a red giant is interrupted, due either to Roche lobe overflow onto a binary companion or to envelope ejection following a common-envelope phase in a tidal-capture binary. Alternatively, they could be very low mass core-He-burning stars. Both the CVs and the new class of faint UV stars are strongly concentrated toward the cluster center, to the extent that mass segregation from two-body relaxation alone may be unable to explain their distribution.
\end{abstract}

Subject headings: binaries: close — globular clusters: individual (NGC 6397) —

Hertzsprung-Russell diagram — novae, cataclysmic variables - white dwarfs

\section{INTRODUCTION}

The center of NGC 6397 is a prime region in which to investigate the effects of stellar interactions. At just $2.2 \mathrm{kpc}$, its high-density core can be studied in detail: with the Hubble Space Telescope (HST), stars well below the turnoff can be observed all the way to the cluster center. The cluster's structure has been investigated most recently by Sosin (1997), who finds within the power-law cusp a resolved core with a radius of $\sim 5^{\prime \prime}$. Fokker-Planck models constructed by Dull (1996) match the strong mass segregation seen by King, Sosin, \& Cool (1995) and put the central density at $\sim 6 \times 10^{7} \mathcal{M}_{\odot} \mathrm{pc}^{-3}$. Dark remnants in the form of neutron stars and/or massive white dwarfs appear to make up 30\% of the cluster mass (Dull 1996; Drukier 1995).

There is already evidence for activity associated with stellar interactions in NGC 6397. Aurière, Ortolani, \& Lauzeral (1990) suggested that the unusually bright blue stragglers within $r<$ $5^{\prime \prime}$ of the cluster center could be collisional mergers or collisionally hardened primordial binaries. Hardened primordial binaries were also put forward by Djorgovski et al. (1991) as a potential explanation for the central deficit of red giants. Three candidate cataclysmic variables $(\mathrm{CVs})$, the possible products of tidal capture (see, e.g., Di Stefano \& Rappaport 1993), were found within $\sim 10^{\prime \prime}$ of the cluster center, using $\mathrm{H} \alpha$ imaging with the HST Wide Field Planetary Camera 1 (WFPC1; Cool et al.

\footnotetext{
${ }^{1}$ Based on observations with the NASA/ESA Hubble Space Telescope, obtained at the Space Telescope Science Institute, which is operated by AURA, Inc., under NASA contract NAS 5-26555.
}

1995) and were confirmed spectroscopically with the HST Faint Object Spectrograph (FOS; Grindlay et al. 1995). Two other faint UV stars near the center were identified as $\mathrm{CV}$ candidates with the HST Faint Object Camera (De Marchi \& Paresce 1994; Cool et al. 1995) but were too faint for the WFPC1 $\mathrm{H} \alpha$ study.

Here we present results of an HST/WFPC2 study of the center of NGC 6397, preliminary results of which have appeared in Cool (1997) and Sosin (1997). This Letter will focus on the cluster's faint UV stars, presenting light curves, magnitudes, and broadband colors for the five previously known objects, together with two newly discovered objects. A companion paper (Edmonds et al. 1998, hereafter EG98) presents our $H S T / F O S$ results for these objects. Results concerning main-sequence stars, the white dwarf sequence, and detached binaries will appear elsewhere. We describe the data and results in $\S 2$ and discuss the findings in $\S 3$.

\section{OBSERVATIONS AND RESULTS}

On 1996 March 6-7, we observed NGC 6397 using the WFPC2, with the PC1 chip approximately centered on the cluster core. A total of 55 400 s exposures were taken spanning $\sim 16 \mathrm{hr}$. The first 35 exposures used the F336W $\left(U_{336}\right)$ filter, and the remaining 20 used the F439W ( $\left.B_{439}\right)$ filter. Shorter exposures were also taken in these filters and in the $\mathrm{F} 555 \mathrm{~W}$ $\left(V_{555}\right)$ and F814W $\left(I_{814}\right)$ filters. The STScI "pipeline" calibration procedures were applied to all the images. Sets of co-aligned $U_{336}$ and $B_{439}$ images were stacked to produce deep images that are free of cosmic rays at each pointing. Photometry was carried out on the combined images using the "weighted, subtracted 


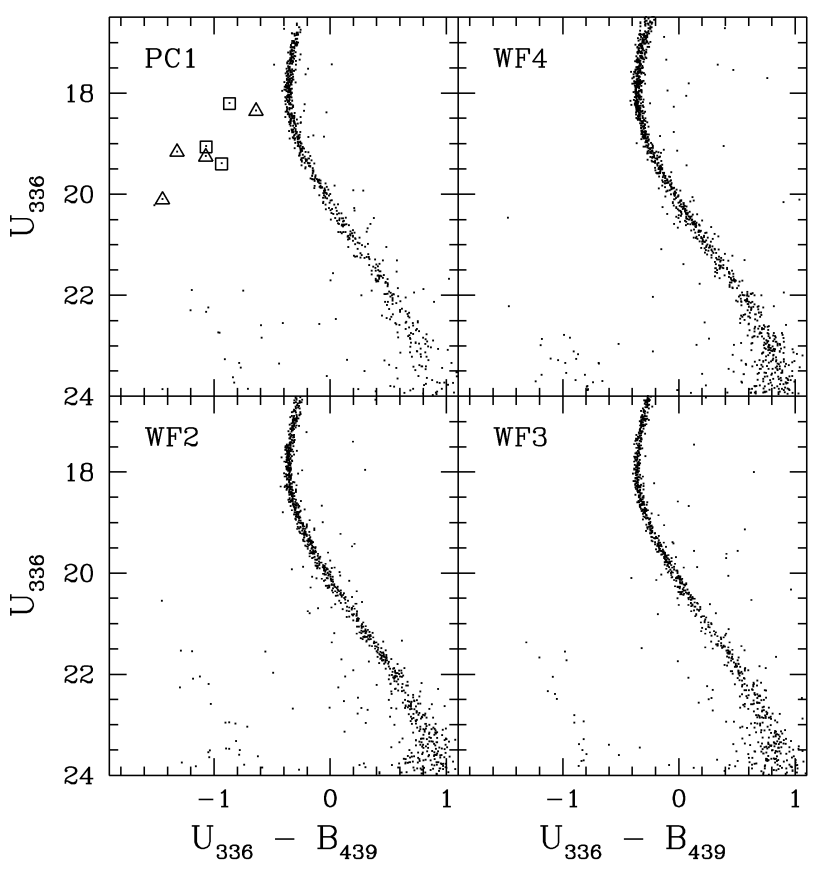

FIG. 1.-CMDs for the central regions of NGC 6397, divided into stars appearing in each of the four WFPC2 chips. The stars marked with triangles are CVs. The stars marked with squares are a newly identified class of nonvariable faint UV stars that may be helium white dwarfs.

aperture photometry" method described by Cool \& King (1995). Instrumental magnitudes were transformed to the WFPC2 "synthetic system" using the prescriptions in Holtzman et al. (1995). Details will appear in a future paper (Cool et al. 1998).

The $U_{336}-B_{439}$ versus $U_{336}$ color-magnitude diagrams (CMDs) are shown in Figure 1. The bend in the upper main sequence is a filter effect; the turnoff is off the top edge of the diagrams. Stars to the left and right of the main sequence are largely field stars, owing to the low Galactic latitude of NGC $6397\left(b=-11^{\circ}\right)$. The upper end of a white dwarf (WD) sequence is also visible in the bottom left-hand corner of each panel. The stars of interest here are marked with squares and triangles. All seven appear in the PC1 chip. The absence of any stars in the WF chips in the corresponding region of the CMD implies that these seven stars are cluster members, given the much larger combined area of the WF chips relative to the PC1 (a factor of $\sim 14$ ). Moreover, the stars are centrally concentrated within the cluster: each of the four chips contains a similar number of cluster stars, yet these UV-bright stars are all on the PC1 chip. Five of the seven have been identified in previous HST studies as emission-line stars and/or as UV-bright stars (Grindlay et al. 1995 and references therein). The two new UV-bright stars identified here were either outside of the field of view or below the magnitude limit of previous studies.

Light curves for the seven UV-bright stars and three nonvariable reference stars are presented in Figure 2. Four of the UV-bright stars are variables. The three previously known CVs (CVs 1-3) all show variability on timescales of hours, as well as the shorter timescale variability known as "flicker." The flicker is most evident for the faintest of the three (CV 3) but can be seen even in the brightest (CV 1), when compared with a nonvariable reference star of similar magnitude (REF 1). The fourth variable star, whose light curve is very similar to that of CV 3, we identify as a new candidate CV (CV 4 in Fig. 2);

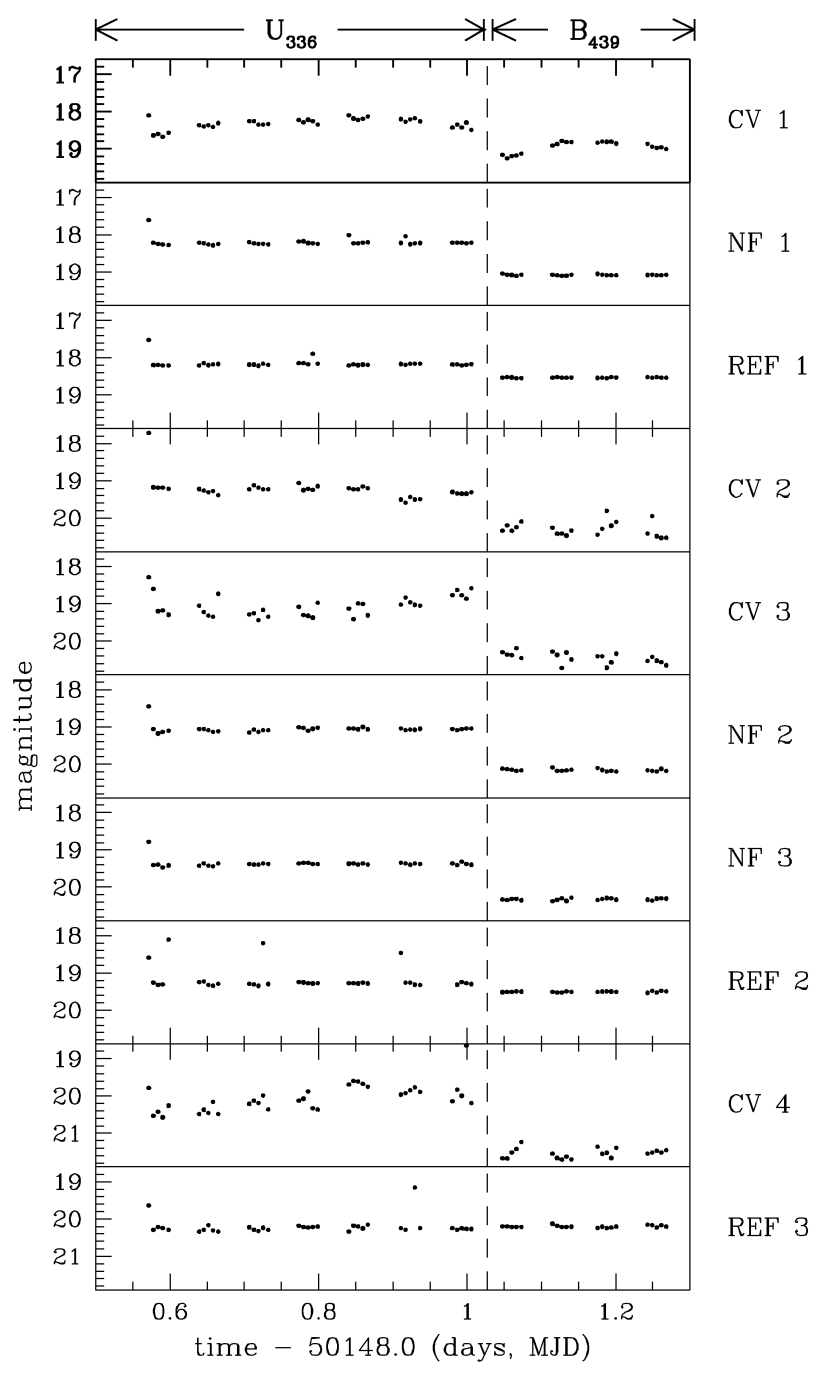

FIG. 2.-Light curves for the seven faint UV stars near the center of the cluster, along with three nonvariable reference stars. The change of filter from $U_{336}$ to $B_{439}$ part way through the observations is represented by a dashed vertical line. The occasional high points (e.g., 1-4 per light curve) are due to cosmic rays. The reference stars provide a baseline for comparison of the UV star light curves. All four CVs are clearly variable, while the three "NFs" have light curves that are very similar to the nonvariable reference stars.

follow-up HST/FOS spectroscopy (EG98) reveals Balmer, He I, and He II emission lines, confirming it as a CV. These flickering variables are marked with triangles in Figure 1. The new $\mathrm{CV}$ is the faintest of the four and was just beyond the detection limit in our WFPC1 $\mathrm{H} \alpha$ study (Cool et al. 1995).

In contrast to the CVs, the UV-bright stars marked with squares in Figure 1 show no sign of photometric variability. We refer to them hereafter as "nonflickerers" (NFs) and have labeled them NFs 1-3 in Figure 2. The rms variations in the light curves of these stars are typical of other nonvariable stars of similar apparent magnitude (cf. REFs 1-3).

In Figure 3, we compare the broadband colors of the CVs versus the NFs. In the left panel $\left(U_{336}-B_{439}\right)$, all seven stars lie to the left of the main sequence. In the central panel $\left(B_{439}-V_{555}\right)$, the brightest two CVs have shifted onto the main sequence, while the fainter two have moved to the red side of the three NFs. The separation between flickerers and nonflickerers becomes even more distinct in the right-hand panel $\left(V_{555}-I_{814}\right)$. Here all the $\mathrm{CVs}$ are either on or very close to 


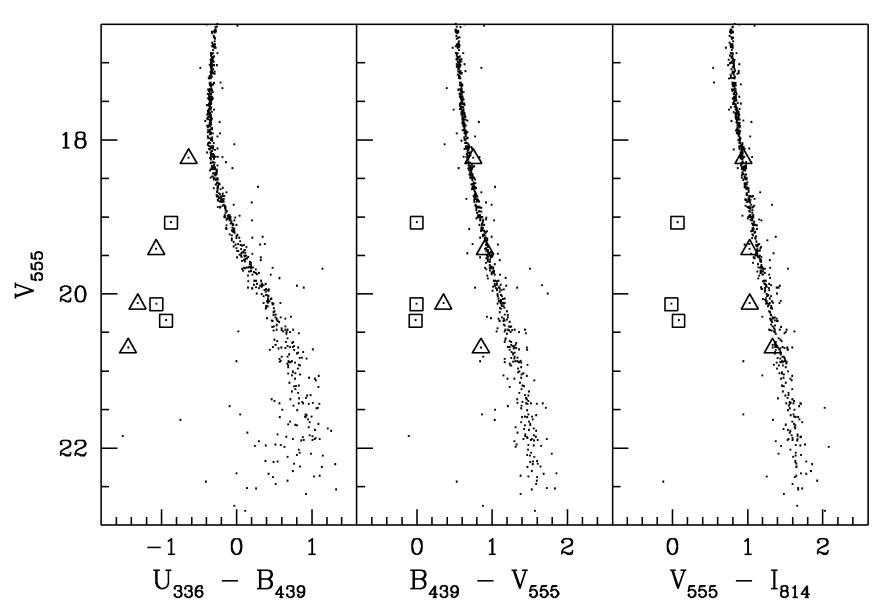

FIG. 3.-Three CMDs for stars in the PC1 chip. The symbols are the same as in Fig. 1. Note the differing behavior of the NFs as compared with the CVs as one moves from the bluest to the reddest color index.

the main sequence. In contrast, the NFs remain well to the blue side of the main sequence in every filter combination. In short, the three NFs are simply blue stars, whereas the four CVs have UV excesses. Table 1 lists positions, mean absolute magnitudes, and dereddened colors of the seven faint UV stars, together with their radial offsets from the cluster center and a cross reference to the identification numbers used by Cool et al. (1995).

\section{DISCUSSION}

The NFs appear to constitute a distinct class of faint UV stars in the cluster. Their broadband colors and lack of photometric variability both set them apart from the CVs. In addition, two of the three, while close to the cluster center, are outside the error circles of the three central X-ray sources detected with ROSAT (Cool et al. 1993). This is in contrast to the four CVs, all of which are within the ROSAT HRI X-ray error circles. Thus, NGC 6397 contains two distinct populations of centrally concentrated faint UV stars. Below we explore what can be learned about the nature and characteristics of the $\mathrm{NFs}$ and CVs from their magnitudes, colors, numbers, and spatial distribution within the cluster.

The first possibility to consider is that the NFs are simply faint "extended horizontal-branch" (EHB) stars, which have been observed in many globular clusters and are thought to be core-helium-burning stars with unusually thin hydrogen envelopes. However, EHB stars are expected to have $M_{V} \sim 4-5$ even when stripped of nearly their entire hydrogen envelopes
(Dorman 1992), more than 2 mag brighter than the NFs. Moreover, NGC 6397 shows no sign of a faint blue horizontal-branch (HB) extension (Lauzeral et al. 1992). If the NFs are Heburning stars, then their masses would need to be significantly lower than those of the cluster's HB stars. A scenario in which low-mass core-He-burning stars may be produced from the merger of two low-mass He WDs has been explored extensively by Iben (1990). With sufficiently small WD progenitor masses, it may be possible to create merged systems with masses well below the HB mass. To explain the NFs, however, it appears that a combination of rather special circumstances would be required to produce exclusively very low mass merger products.

A more promising possibility is that the NFs are low-mass helium white dwarfs. Masses above $\sim 0.3-0.4 \mathcal{M}_{\odot}$ are ruled out, since they are predicted to be too blue at the absolute magnitudes of the NFs (see Cool, Sosin, \& King 1997 for a comparison with the $\log g=7.0-9.0$ WD models of Bergeron, Wesemael, \& Beauchamp 1995). Below this mass, the expected colors of WDs are consistent with those of the NFs. EG98 report HST/FOS spectroscopy of NF 2 showing that it has log $g \simeq 6.25 \pm 1.0$ and a continuum temperature consistent with the values $\log T_{\text {eff }} \simeq 4.3 \pm 0.1$ and $B C_{V} \simeq 2.0 \pm 0.2$ derived here from our measured $(B-V)_{0}$ value. The implied luminosities are $\log L / L_{\odot} \simeq 0.1$ to -0.6 . In the helium WD models of Althaus \& Benvenuto (1997), these values correspond to $\mathcal{M} \simeq 0.25 \mathcal{M}_{\odot}$ and $\log g \simeq 6.0-6.7$, which are consistent with the spectroscopic results. Detailed comparisons with He WDs models, evolutionary lifetimes, and a discussion of the apparent lack of fainter He WDs in Figure 1 are given by EG98.

If indeed the NFs are $\sim 0.25 \mathcal{M}_{\odot} \mathrm{He}$ WDs, we need to consider the constraints on the formation rate of such systems in NGC 6397. To form a He WD, the envelope of a red giant branch (RGB) star must somehow be removed before the first ascent of the branch is complete. Two mechanisms by which this might be accomplished are as follows: (1) Roche lobe overflow onto a binary companion (Webbink 1975) and (2) a collision that produces a common-envelope system, followed by envelope ejection (Davies, Benz, \& Hills 1991). In either case, the formation rate is necessarily linked to post-mainsequence evolution. An order-of-magnitude estimate of the fraction of RGB stars that would need to be stripped can be made by noting that there are six HB stars within the PC1 chip where the three NFs reside. While the lifetimes of He WDs are uncertain (see, e.g., Iben \& Tutukov 1986), if we assume that they are similar to those of HB stars $\left(10^{8} \mathrm{yr}\right)$, then $\sim \frac{1}{3}$ of all RGB stars would need to be stripped. If binaries alone were responsible for the stripping, the required $\frac{1}{3}$ binary fraction would be compatible with binary fractions in the range of

TABLE 1

FAINT UV STARS IN NGC 6397

\begin{tabular}{|c|c|c|c|c|c|c|c|c|}
\hline Star & $x$ & $y$ & $M_{V}$ & $(U-B)_{0}$ & $(B-V)_{0}$ & $(V-I)_{0}$ & $\begin{array}{c}\Delta r \\
(\operatorname{arcsec})\end{array}$ & $\begin{array}{l}\text { Identification } \\
\text { Number }\end{array}$ \\
\hline CV 1. & 451 & 589 & 5.95 & -0.84 & 0.56 & 0.69 & 10.7 & 1 \\
\hline $\mathrm{CV} 2 \ldots \ldots$ & 513 & 349 & 7.13 & -1.27 & 0.72 & 0.77 & 0.9 & 2 \\
\hline CV $3 \ldots \ldots$ & 314 & 436 & 7.84 & -1.51 & 0.16 & 0.78 & 9.9 & 3 \\
\hline $\mathrm{CV} 4 \ldots \ldots$ & 454 & 525 & 8.41 & -1.65 & 0.67 & 1.08 & 7.8 & New \\
\hline NF $1 \ldots \ldots$ & 584 & 717 & 6.79 & -1.06 & -0.20 & -0.18 & 16.4 & New \\
\hline NF $2 \ldots \ldots$ & 647 & 551 & 7.84 & -1.27 & -0.19 & -0.27 & 10.3 & 7 \\
\hline NF $3 \ldots \ldots$ & 442 & 303 & 8.06 & -1.13 & -0.21 & -0.17 & 4.6 & 6 \\
\hline
\end{tabular}

Note. - Star coordinates refer to archive exposure u33r0101t. For the conversions to absolute magnitudes, we adopted $(m-M)_{0}=11.71, A_{U}=0.97, A_{B}=0.77, A_{V}=0.58$, and $A_{I}=0.33$. Radial offsets are based on a cluster center at $(x, y)=(529,378)$, following Sosin 1997. 
$10 \%-40 \%$ that have been suggested (Mateo 1996 and references therein). Binaries that reside in the core are likely to be sufficiently hardened as to make Roche lobe overflow inevitable (Hut et al. 1992). Binaries formed by tidal capture could also contribute to the pool of He WD progenitors. If, on the other hand, stellar interactions were the sole production route for He WDs, the required collision rate for RGB stars would be about $3 \times 10^{-8} \mathrm{yr}^{-1}$. This is an order of magnitude higher than the rate at which common-envelope systems form via collisions between single stars in NGC 6397, as estimated by Davies (1992). However, it is compatible with the rates he computed for binary-single-star encounters. Thus, binary evolution and/or collisions involving binaries seem to provide at least plausible mechanisms for forming He WDs. Whatever the production mechanism, the existence of He WDs would provide an important link to the apparent deficit of RGB stars near the cluster center (Djorgovski et al. 1991).

Whether He WDs form from preexisting binaries or via collisionally formed binaries, they ought to have binary companions at present. We can place limits on the mass of any mainsequence companion by assuming that the $B_{439}-V_{555}$ colors are due exclusively to a He WD and by asking whether the $V_{555}-I_{814}$ colors are compatible with a single-temperature model. We find that they are and that companions with $\mathcal{M} \gtrsim 0.15 \mathcal{M}_{\odot}$ can be ruled out. Fainter main-sequence stars, or dark companions in the form of cool WDs or neutron stars, are of course allowed. We note that massive remnants would be a natural result of either formation mechanism since they appear to be the most common species within the central few arcseconds of the cluster (Dull 1996), where collisions and/or exchanges into primordial binaries are likely to occur.

Mass estimates for the CV secondaries can also be gleaned from the present data. The locations of the CVs on or near the main sequence in the right-hand panel of Figure 3 suggest that the secondaries dominate in $V_{555}$ and $I_{814}$. By assuming that the disks make a negligible contribution in $I_{814}$, we deduce masses of $\sim 0.69,0.56,0.48$, and $0.44 \mathcal{M}_{\odot}$ for the secondary stars in CVs $1,2,3$, and 4 , respectively, using a mass- $M_{I}$ relation for stars with $[\mathrm{Fe} / \mathrm{H}]=-1.9$ kindly provided by $\mathrm{E}$. Brocato and based on the models by Alexander et al. (1997). Evolutionary effects, and thus departures from apparent main-sequence masses, appear to be small for CVs with orbital periods less than $7 \mathrm{hr}$ (Smith \& Dhillon 1998), as is likely the case for these cluster CVs (EG98); in any case, the proximity of the $\mathrm{CVs}$ to the main sequence in $V_{555}-I_{814}$ suggests that they are unevolved. That all four secondaries are drawn from the upper half of the mass range of the main sequence is interesting, considering that $\mathrm{CVs}$ with less massive secondaries should have been observable (see Fig. 1). Such a preponderance of massive secondaries would be expected if the CVs form via stellar interactions. However, deeper and more systematic CV searches are needed before the significance of this can be properly assessed. At present, we simply note that, relative to field CVs, the secondaries are rather bright (owing to a combination of low metallicity and high mass) and that the disks are rather faint $\left(M_{V} \sim 8-10.5\right.$, when secondaries are subtracted out). Disks this faint are not unusual among field CVs, but that all four should be so faint has interesting implications for their nature (EG98).

Like the bright blue stragglers (BSs), both the CVs and the NFs are strongly concentrated toward the cluster center. This effect is expected due to mass segregation, since these objects are more massive than typical cluster members. However, the strength of the central concentration is hard to understand. All 13 of the relevant objects (bright BSs, CVs, and NFs) lie within $16^{\prime \prime}$ of the cluster center. For comparison, in Fokker-Planck models of NGC 6397 (Dull 1996), only $40 \%$ of the neutron stars fall within this radius. Binary stars and merger products are unlikely to be more massive than twice the main-sequence turnoff mass, so the typical mass of these exotic objects should be similar to the $1.4 \mathcal{M}_{\odot}$ assumed for the neutron stars. Thus, either these unusual objects are much more massive than we expect (as may be the case for the brightest BS according to Sills \& Bailyn 1998) or some process other than mass segregation via two-body relaxation is at work. The strong central concentration provides compelling evidence that the formation of CVs, NFs, and bright BSs is linked to stellar interactions, which are strongly favored within $5^{\prime \prime}$ of the cluster center.

The existence within a single cluster of three distinct classes of stars, each of whose formation is likely to be tied to stellar interactions, presents an opportunity to test the outcomes of these rare but astrophysically important events. Constraints on the numbers and radial distributions of various stellar species in globular clusters are steadily improving thanks in part to star counts provided by $H S T$ and increasingly sophisticated dynamical modeling. A reevaluation of stellar interaction rates that incorporates these new developments would be valuable. A census of faint UV stars in other globular clusters would also be of interest, particularly if carried out in a manner that clearly distinguishes between CVs and NFs. The distinction can be made on the basis of $\mathrm{H} \alpha$ imaging, multicolor broadband photometry, and/or sensitive variability studies.

We thank P. Edmonds, B. Dorman, I. King, R. Saffer, A. Sarajedini, and C. Sosin for discussions and E. Brocato for providing isochrones for NGC 6397. This work was supported in part by NASA grant HST-GO-5929.

\section{REFERENCES}

Alexander, D. R., Brocato, E., Cassisi, S., Castellani, V., Ciacio, F., \& Degl'Innocenti, S. 1997, A\&A, 317, 90

Althaus, L. G., \& Benvenuto, O. G. 1997, ApJ, 477, 313

Aurière, M., Ortolani, S., \& Lauzeral, C. 1990, Nature, 344, 638

Bergeron, P., Wesemael, F., \& Beauchamp, A. 1995, PASP, 107, 1047

Cool, A. M. 1997, in Advances in Stellar Evolution, ed. R. T. Rood \& A. Renzini (Cambridge: Cambridge Univ. Press), 191

Cool, A. M., et al. 1998, in preparation

Cool, A. M., Grindlay, J. E., Cohn, H. N., Lugger, P. M., \& Slavin, S. D. 1995, ApJ, 439, 695

Cool, A. M., Grindlay, J. E., Krockenberger, M., \& Bailyn, C. D. 1993, ApJ, 410, L103

Cool, A. M., \& King, I. R. 1995, in Calibrating Hubble Space Telescope: PostServicing Mission, ed. A. Koratkar \& C. Leitherer (Baltimore: STScI), 290
Cool, A. M., Sosin, C., \& King, I. R. 1997, in White Dwarfs, ed. J. Isern, M. Hernanz, \& E. Garcia-Berro (Dordrecht: Kluwer), 129

Davies, M. B. 1992, Ph.D. thesis, Harvard Univ.

Davies, M. B., Benz, W., \& Hills, J. G. 1991, ApJ, 381, 449

De Marchi, G., \& Paresce, F. 1994, A\&A, 281, L13

Di Stefano, R., \& Rappaport, S. 1993, ApJ, 423, 274

Djorgovski, S., Piotto, G., Phinney, E. S., \& Chernoff, D. F. 1991, ApJ, 372, L41

Dorman, B. 1992, ApJS, 81, 221

Dull, J. D. 1996, Ph.D. thesis, Indiana Univ.

Drukier, G. A. 1995, ApJS, 100, 347

Edmonds, P. D., Grindlay, J. E., Cool, A. M., Cohn, H. N., Lugger, P. M., \& Bailyn, C. D. 1998, ApJ, submitted (EG98)

Grindlay, J. E., Cool, A. M., Callanan, P. C., Bailyn, C. D., Cohn, H. N., \& Lugger, P. M. 1995, ApJ, 455, L47 
Holtzman, J. A., Burrows, C. J., Casertano, S., Hester, J. J., Trauger, J. T., Watson, A. M., \& Worthey, G. 1995, PASP, 107, 1065

Hut, P., et al. 1992, PASP, 104, 981

Iben, I. 1990, ApJ, 353, 215

Iben, I., \& Tutukov, A. V. 1986, ApJ, 311, 742

King, I. R., Sosin, C., \& Cool, A. M. 1995, ApJ, 452, L33

Lauzeral, C., Ortolani, S., Aurière, M., \& Melnick, J. 1992, A\&A, 262, 63
Mateo, M. 1996, in ASP Conf. Ser. Vol. 90, The Origins, Evolution, and Destinies of Binary Stars in Clusters, ed. E. F. Milone \& J.-C. Mermilliod (San Francisco: ASP), 21

Sills, A., \& Bailyn, C. D. 1998, in preparation

Smith, D. A., \& Dhillon, V. S. 1998, MNRAS, in press

Sosin, C. A. 1997, Ph.D. thesis, UC Berkeley

Webbink, R. F. 1975, MNRAS, 171, 555 